\title{
Comparison of antenatal care factors and pregnancy outcome in rural and urban context
}

\author{
Irisa Zile ${ }^{1, *}$, Dace Rezeberga ${ }^{2}$, Gunta Lazdane $^{3}$, and Iveta Gavare ${ }^{1}$ \\ ${ }^{1}$ The Centre for Disease Prevention and Control of Latvia, Riga, Latvia \\ ${ }^{2}$ Rīga Stradinš̌ University, Department of Obstetrics and Gynaecology, Riga, Latvia \\ ${ }^{3}$ Rìga Stradiņš University, Institute of Public Health, Riga, Latvia
}

\begin{abstract}
Antenatal care plays a basic role in the management of woman's health during pregnancy, and women who have not been registered to antenatal care are at increased risk of poor pregnancy outcome, including stillbirth. The aim of this study was to identify differences in maternal characteristics, antenatal care factors and perinatal mortality between urban and rural area in Latvia. The Medical Birth Register of Latvia (MBR) was used as a data source, all births in $2017(n=20,406)$ were included in the analysis. Mother's place of residence was categorized in 3 groups: Riga, other cities and rural area. Higher risk of smoking $(\mathrm{OR}=1.4 ; p<0.001)$, late first antenatal visit (after 12th gestation week) $(\mathrm{OR}=1.2 ; p<0.05)$ and incomplete antenatal care (including cases without care) $(\mathrm{OR}=1.3$; $p<0.05$ ) were detected more in other regions (other cities and rural area) than in Riga. After adjustment by maternal age and education, just late first antenatal visit was significantly higher in other regions $(\mathrm{ORadj}=1.2$; $p<0.05)$ vs. Riga. New-born mortality in perinatal period is substantially higher in other regions vs. Riga. The results indicate health education gap and potential inequalities in receiving antenatal care and emergency obstetric care by regions.
\end{abstract}

Key words: antenatal care; rural; urban; pregnancy outcome.

\section{Introduction}

Antenatal care is defined as the routine care of pregnant women provided between conception and the onset of labour. Antenatal care is an opportunity to provide care for prevention and management of existing and potential causes of maternal and new-born mortality and morbidity [1].

Women who have not been registered to antenatal care are at increased risk of poor pregnancy outcomes, including stillbirth [1-4]. According to statistical data in the general population in $2017,0.8 \%$ of live births occurred without antenatal care, but the rate of stillbirths that occurred without prenatal care was 8 times higher, at 6.9\% [5].

Literature data show that the place of residence, urban or rural area, is also a factor affecting the use of antenatal care. There are differences between the urban and rural areas in knowledge, attitude, and practices of women towards antenatal care [6-8].

There is a lack of sufficient epidemiologically based data about this issue in Latvia. The use of antenatal care varies within the country, proportion of women with timely first antenatal visit (before the 12th gestational week) differs from $97 \%$ in Riga to $83 \%$ in Kurzeme region,

\footnotetext{
* Corresponding author: irisa.zile@spkc.gov.lv
}

(C) The Authors, published by EDP Sciences. This is an Open Access article distributed under the terms of the Creative Commons Attribution License 4.0 (http://creativecommons.org/licenses/by/4.0/). 
an average in Latvia - 93\% [5]. The aim of this study was to identify differences in maternal characteristics, antenatal care factors and perinatal mortality between urban and rural areas in Latvia.

\section{Material and methods}

The Medical Birth Register of Latvia (MBR) was used as data source. All births in 2017 $(n=20,406)$ were included in the analysis. The study analysed and compared antenatal care factors and pregnancy outcomes between urban and rural areas. Mother's place of residence was categorized into 3 groups: Riga, other Latvian biggest cities and rural areas (i.e., counties).

Information about the evaluation of antenatal care was based on the timing of the first antenatal visit and completeness. Antenatal care model in Latvia recommends that the first antenatal care visit takes place within the first trimester (i.e., gestational age of $<12$ weeks) and eight contacts with a gynaecologist throughout pregnancy [9]. Antenatal care is complete if several conditions are covered: first antenatal visit till 12th week of pregnancy and obligatory antenatal visits with all necessary antenatal checks and tests as per guidelines (blood, urine tests; ultrasound screening; genetic tests; etc.) and incomplete care was defined if one or more conditions are missing, e.g., late first antenatal visit or less antenatal visits or checks. Low birth weight (LBW) has been defined as the first weight recorded within hours of the birth of $<2500$ grams.

The descriptive statistics for all continuous variables are reported as mean and standard deviation. Categorical data are reported as percentages and $95 \%$ confidence interval (CI) and Chi-square test for comparing categorical variables were used. The odds ratios (OR) were calculated (adjusted and non-adjusted) and 95\% CI were computed to measure the strength of the association between the outcome and the explanatory variables.

Place of residence was merged into 2 groups for OR analysis: Riga and other regions (other cities and rural regions).

\section{Results}

The study analysed data on 20,406 births in 2017. 34.5\% (95CI 33.8-35.1) ( $n=7030)$ birth took place in Riga, $15.3 \%$ (95\%CI 14.8-15.8) $(n=3121)$ other cities and 50.3\% (95\%CI 49.6-51.0) $(n=10,255)$ in rural regions. The average mother's age in Riga was higher 31 years than in other cities and rural area $29(p<0.001)$. Younger mothers $(\leq 20)$ were more often in rural regions $(p<0.001)-6.2 \%$ (95\%CI 5.7-6.7) $(n=634)$ comparing with Riga 2.5\% (95\%CI 2.2-3.0) ( $n=179)$. Higher education level was in Riga ( $p<0.001)$, by 20 percentage points higher than in rural regions (65\% to $44.4 \%)$. There are no differences between regions in average gestational age and birth weight of new-borns, although a higher proportion of new-borns with low birth weight $(\leq 2499 \mathrm{~g})$ was observed $(p<0.01)$ in rural regions $4.6 \%(95 \% \mathrm{CI} 4.2-5.1)(n=476)$ than in Riga and other cities. The characterization of parameters by region is shown in Table 1 .

Higher proportion $(p<0.05)$ of mothers with incomplete antenatal care was outside Riga $(5.4 \% ; 95 \%$ CI $4.9-6.0 ; n=377)-$ in other cities $6.4 \%(95 \%$ CI $5.6-7.3)(n=200)$ and rural area $6.6 \%(95 \%$ CI $6.2-7.1)(n=681)$ as well as with late first antenatal visit $(p<0.001)-$ rural regions 6.4\% (95\%CI 6.0-6.9) $(n=653)$ against 5.3\% (95\%CI 4.8-5.8) $(n=370)$ in Riga. US in the 1st trimester an average were $77.4 \%$ (95\% CI 77.0-78.1) and in the 2nd trimester $-93.4 \%$ (95\% CI 93.1-93.8), there are no differences between regions.

In relation to such lifestyle factors as smoking, differences were observed by regions - a higher proportion was in other cities and rural areas than in Riga, an average by 2 percentage 
Table 1. Characterization of parameters by region.

\begin{tabular}{|c|c|c|c|c|}
\hline Characteristics & $\begin{array}{c}\text { Riga } \\
(n=7030)\end{array}$ & $\begin{array}{c}\text { Other cities } \\
(n=3121)\end{array}$ & $\begin{array}{c}\text { Rural regions } \\
\text { (10255) }\end{array}$ & $p$ value \\
\hline Mean maternal age ${ }^{1}$ & $31( \pm 5.2)^{\dagger}$ & $29( \pm 5.7)$ & $29( \pm 5.7)$ & $<0.01$ \\
\hline Mean birth weight $(\mathrm{g})^{1}$ & $3515.7( \pm 587)$ & $3519.1( \pm 587)$ & $3485.3( \pm 603)$ & NS \\
\hline \multicolumn{5}{|l|}{ Maternal age group ${ }^{2}$ : } \\
\hline$\leq 20$ years & $2.5(179)^{\dagger}$ & $5.9(185)$ & $6.2(634)$ & $<0.001$ \\
\hline $21-34$ years & $74.0(5199)$ & $75.6(2359)$ & $75.0(7695)$ & NS \\
\hline$\geq 35$ years & $23.5(1652)^{\dagger}$ & $18.5(577)$ & $18.8(1926)$ & $<0.001$ \\
\hline \multicolumn{5}{|l|}{ Maternal education ${ }^{2}$ : } \\
\hline elementary & $7.5(525)^{\dagger}$ & $13.5(422)$ & $14.6(1500)$ & $<0.001$ \\
\hline secondary & $27.6(1938)^{\dagger}$ & $40.2(1256)$ & $41.0(4204)$ & $<0.001$ \\
\hline higher & $65.0(4567)^{\dagger}$ & $46.2(1443)$ & $44.4(4551)$ & $<0.001$ \\
\hline \multicolumn{5}{|l|}{ Parity ${ }^{2}$ : } \\
\hline Primipara & $44.4(3120)^{\dagger}$ & $37.8(1181)$ & $38.7(3971)$ & $<0.001$ \\
\hline Low birth weight ${ }^{2}$ & $3.8(270)$ & $3.5(110)$ & $4.6(476)^{\dagger \dagger}$ & $<0.01$ \\
\hline Maternal smoking ${ }^{2}$ & $5.7(398)^{\dagger}$ & $8.4(263)$ & $7.5(766)$ & $<0.001$ \\
\hline \multicolumn{5}{|l|}{ Antenatal care ${ }^{2}:$} \\
\hline Late fist antenatal visit & $5.3(370)$ & $5.1(159)$ & $6.4(653)^{\dagger \dagger}$ & $<0.01$ \\
\hline Incomplete antenatal care & $5.4(377)^{\dagger}$ & $6.4(200)$ & $6.6(681)$ & $<0.01$ \\
\hline Without antenatal care & $0.6(42)$ & $0.4(14)$ & $1.1(110)^{\dagger \dagger}$ & $<0.001$ \\
\hline
\end{tabular}

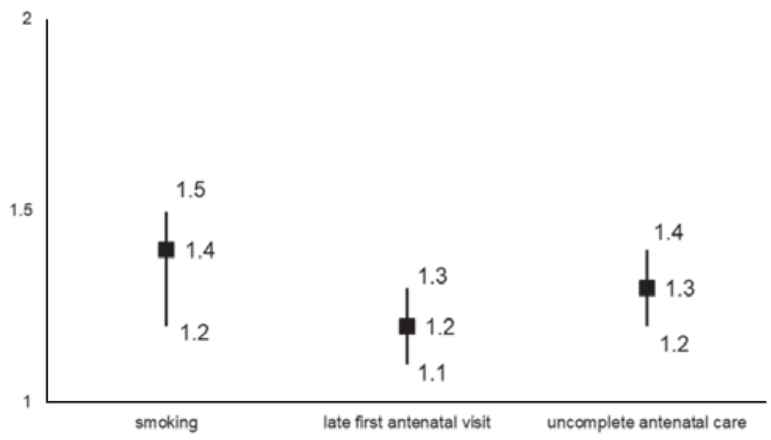

Figure 1. Odds ratio of different factors in other regions (other cities and rural area) vs. Riga, based on univariate analysis.

points $(p<0.001)$. An average obesity was detected in 3.5\% (95\% CI 3.3-3.8) $(n=723)$, there are no differences between regions.

Higher risk of smoking $(\mathrm{OR}=1.4 ; p<0.001)$, late first antenatal visit (after 12th gestation week) $(\mathrm{OR}=1.2 ; p<0.05)$ and incomplete antenatal care (including cases without care) $(\mathrm{OR}=1.3 ; p<0.05)$ were more detected in other regions (other cities and rural area) than in Riga (Fig. 1). After adjustment by maternal age and education, just late first antenatal visit was significantly higher in other regions (ORadj $=1.2 ; p<0.05)$ vs. Riga. 
The gestational week and the birth weight of a new-born are independently associated with an increased risk of perinatal mortality in all regions.

New-born mortality in the perinatal period is substantially higher in other regions than in Riga (ORadj $=1.5 ; p<0.05)$. OR was adjusted by maternal age, gestational week, birth weight and parity. Adjusted by maternal education, perinatal mortality OR is still slightly higher $(\mathrm{ORadj}=1.3 ; p>0.05)$ in other regions vs. Riga but not more statistically significant. Mother education also as an independent factor was associated with increased perinatal mortality $(\mathrm{OR}=2.1 ; p<0.01)$.

\section{Discussion}

Antenatal care plays a basic role in the management of a woman's health during pregnancy. An early antenatal care visit allows providing screening and tests that are most effective in early stages of the pregnancy.

The procedure of antenatal care as well as during and after childbirth is determined by the Cabinet of Ministers Regulation No. 611 "Procedures for providing childbirth assistance" [9]. Prenatal care in Latvia is provided by a gynaecologist (obstetrician), a midwife or family doctor if the pregnancy is physiological. The health care services, which have agreed with the National Health Service (NHS), shall provide state-funded health care services. Pregnant women do not have to pay patient co-payments for health care services related to pregnancy and post-natal monitoring and pregnancy with a specialist contracted with NHS, as well as childbirth in a hospital with NHS is a state-funded service.

In this study, the proportion of timely first antenatal visit an average was $94.2 \%$. For example, in the United States $77.1 \%$ of women who gave birth in 2016 initiated prenatal care in the first trimester of pregnancy [10].

The estimated worldwide coverage of early antenatal care visits increased from $40.9 \%$ in 1990 to $58.6 \%$ in 2013, corresponding to a $43.3 \%$ increase. Overall coverage in the developing regions was $48.1 \%$ in 2013 compared with $84.8 \%$ in the developed regions. In 2013 , the estimated coverage of early antenatal care visits was $24.0 \%$ in low-income countries compared with $81.9 \%$ in high-income countries [1].

The Childbirth and Health Study in Iceland indicates that antenatal care can play an important role in preparing women for birth. The majority (87\%) of the women want to be informed about birth in the antenatal phase of care, and 41\% reported 5-6 months postpartum that too little time had been spent on this issue by health care professionals. Women who experienced their birth as difficult or very difficult were more likely to report that insufficient time had been spent on information than women who had experienced their birth as easy or very easy [11].

Many studies have identified socio-demographic predictors of late initiation of antenatal care $[1,6-8,10,12,13]$. This study also demonstrates variation in the timely and complete antenatal care receipt by place of residence. Higher risk of late first antenatal visit and incomplete antenatal care were observed in rural regions. An average $6 \%$ of received incomplete antenatal care. Study in the United States observed a higher proportion $-15 \%$ [10]. Survey data from England has found that the proportion of pregnant women having their booking appointment by 12 completed weeks of pregnancy is $86 \%$ and by 10 weeks of pregnancy is $63 \%$ [14]. In the United Kingdom, the timing of the antenatal booking appointment is used as an indicator of access to care in pregnancy and antenatal care ought to be promoted as woman-centred and as means to empower women to feel in control of their pregnancy [14].

To improve health outcomes and reduce disparities among pregnant women and newborns in low- and middle-income countries more should be done to increase access to quality 
maternal health care services for women, especially for those from vulnerable populations [12]. This issue is also very important within the country because from our study results, we can assume that delayed care in rural regions could be related to geographic area and socioeconomic factors. Distance to a healthcare facility could be an important barrier to the use of services, especially in rural areas.

The literature review identifies multiple socio-demographic, reproductive and access related factors that affect the utilization of antenatal care among pregnant women in different countries. Several studies, conducted in different countries, have shown that factors like maternal age, number of living children, education, socioeconomic status, previous bad obstetrical history, support from a spouse, quality of care and distance from health care facility are significantly associated with the use of antenatal care [7].

Another finding of this study is that a higher proportion of smoking during pregnancy was also outside the capital Riga, difference by $2-3$ percentage points. Results of the Australian study showed that among pregnant women who smoked antenatal-care attendance was lower. The same was observed among women who were of younger age, higher parity and unemployed, and not partnered [15].

In some European countries, more than $10 \%$ of women smoke during their pregnancy. A preventive population approach is important to reduce the prevalence of smoking before pregnancy in the childbearing population. The EURO-PERISTAT network has pointed that smoking among pregnant women has declined in high-income countries, but it nonetheless continues to account for a substantial proportion of foetal and infant morbidity and mortality. Smoking before pregnancy and the likelihood of stopping smoking are associated with lower maternal educational level and poverty. Smoking thus contributes to the creation of social inequalities in perinatal health [16].

Literature data show that educated women tend to have a greater awareness of the existence of antenatal care services and the advantages of using such services. It is argued that educated women were more aware of health problems, know more about the availability of health care services, and utilize the information more effectively than non-educated women [7]. Other studies' results also showed that inadequate antenatal care has also been associated with stillbirth, women who had their first antenatal visit after 20 weeks' gestation were slightly more likely to have a stillborn baby than those who had earlier first visits $(\mathrm{OR}=1.12)$ and odds more than tripled with no antenatal care [17].

Our study findings indicated that mother education as an independent factor associated with increased perinatal mortality $(\mathrm{OR}=2.1)$ and it is substantially higher in other regions than in Riga (ORadj=1.5).

Latvian perinatal deaths audit discovered that $19 \%$ of perinatal death cases were related to women who had not taken advantage of antenatal care. Non-attendees were more likely to be smokers $(p<0.001)$ and alcohol abusers $(p<0.005)$, above 35 years of age $(p<0.005)$ and had higher parity $(p<0.001)$. Non-attendees more often had systemic diseases and pregnancy complications. There was no difference in rates of preterm birth and low birth weight between attendees and non-attendees. One-fifth of mothers with perinatal death did not attend antenatal care, and just in some cases when women who attended antenatal care, lack of intervention was related to the perinatal death [18].

The strengths of this work include the Register-based study. Population-based data are essential for planning of health care and for determining temporal trends.

Health of a mother and child is one of the top priorities in Latvia as in worldwide. It should be a priority in the national level that all women have their first antenatal visit by 12 completed weeks of pregnancy, but it is not always of equal priority to women. Lower antenatal care coverage and poor pregnancy outcomes in rural regions detect discrepancies between regions 
and necessity to improve the equality aspects of receiving health care services regardless of the place of residence. The supply of health workers has changed significantly in Latvia and particularly in rural areas. In addition to the absolute shortage and skills imbalance, there is also a strong concentration of health workers in the capital Riga and there is evidence of severe shortages in key specialties (e.g. obstetricians).

Improving maternal and child health and reducing infant mortality are also objectives of the Latvian Policy document "Maternal and Child Health Improvement Plan 2018-2020", developed by the Ministry of Health [19]. The action plan provides for improvements also in screening and maternal and perinatal mortality audit. The action plan foresees changes in the legislative documents to include additional screenings for women and new-borns. Health care standards and guidelines are fundamental requirements for good quality assurance systems. They typically consist of comprehensive, evidence-based clinical guidelines and pathways, which serve as the criteria for quality monitoring activities.

Antenatal care is an opportunity to educate the woman about her health, pregnancy, and childbirth, recognizing danger signs, the benefits of good nutrition and exclusive breastfeeding, the harms of alcohol, tobacco and drugs, and other relevant issues. Latvia has implemented also Maternal Mortality Confidential Analysis conducted by the Expert Committee. The results of this study may deserve attention for policy implementation regarding strategies to improve antenatal and obstetric care and strengthen perinatal audits at the national level.

\section{Conclusions}

A higher proportion of younger mothers, lower education level, smoking during pregnancy and low birth weight are more likely to be in rural regions.

Considerable variation exists in the content of antenatal care by regions. Mothers with incomplete antenatal care, with the late first antenatal visit and smoking more often were observed in other regions vs. Riga, but after adjustment for maternal age and education there is no significant difference, just higher odds of late first antenatal visit in rural regions.

New-born mortality in the perinatal period is substantially higher in other regions vs. Riga.

The results indicate health education gap and potential inequalities in receiving antenatal care and emergency obstetric care in regions.

\section{References}

[1] A. Moller, M. Petzold, D. Chou, L. Say. Early antenatal care visit: a systematic analysis of regional and global levels and trends of coverage from 1990 to 2013. Lancet, 5(10), 977-983 (2017)

[2] J.E. Lawn, H. Blencowe, P. Waiswa, A. Amouzou, C. Mathers, D. Hogan, V. Flenady, J.F. Frøen, Z.U. Qureshi, C. Calderwood, S. Shiekh, F.B. Jassir, D. You, E.M. McClure, M. Mathai, S.E. Cousens. Stillbirths: rates, risk factors, and acceleration towards 2030. Lancent 387(10018), 587-603 (2016)

[3] E.M. McClure, S. Saleem, S.S. Goudar, J.L. Moore, A. Garces, F. Esamai, A. Patel, E. Chomba, F. Althabe, O. Pasha, B.S. Kodkany, C.L. Bose, M. Berreuta, E.A. Liechty, K.M. Hambidge, N.F. Krebs, R.J. Derman, P.L. Hibberd, P. Buekens, A. Manasyan, W.A. Carlo, D.D. Wallace, M. Koso-Thomas, R.L. Goldenberg, Stillbirth rates in lowmiddle income countries 2010-2013: a population-based, multi-country study from the Global Network. Reprod. Health 12(2), S7, (2015) 
[4] M. Aminu, R. Unkels, M. Mdegela, B. Utz, S. Adaji, N. van den Broek. Causes of and factors associated with stillbirth in low- and middle-income countries: a systematic literature review. BJOG 121(4), 141-53 (2014)

[5] The Centre for Disease Prevention and Control of Latvia. Maternal and infant health care. Statistical yearbook of health care in Latvia 19, 258-259 (2017)

[6] A.A. Ewunetie, A.M. Munea, B.T. Meselu, M.M. Simeneh, B.T. Meteku. Delay on first antenatal care visit and its associated factors among pregnant women in public health facilities of Debre Markos town, North West Ethiopia. BMC Pregnancy Childbirth 18, 173 (2018)

[7] S.A. Ali, A.A. Dero, S.A. Ali, G.B. Ali. Factors affecting the utilization of antenatal care among pregnant women: A literature review. J. Preg. Neonatal. Med. 2(2), 41-45 (2018)

[8] J. Amo-Adjei, K. Aduo-Adjei, C. Opoku-Nyamaah, C. Izugbara. Correction: Analysis of socioeconomic differences in the quality of antenatal services in low and middleincome countries (LMICs). PLoS ONE 13(2), e0192513, (2018)

[9] Republic of Latvia Cabinet Regulation No 611 (25.07.2016.) Procedures for providing childbirth assistance

[10] A. Martin, B.E. Hamilton, M.J.K. Osterman, A.K. Driscoll, P. Drake. Births: Final Data for 2016. National vital statistics reports, Division of Vital Statistic 67(1) (2018)

[11] H. Gottfredsdottir, P. Steingrímsdóttir, A. Björnsdóttir, E.Y. Guðmundsdóttir, H. Kristjánsdóttir. Content of antenatal care: Does it prepare women for birth? Midwifery 39, 71-7 (2016)

[12] J. Sharma, M. O'Connor, R.R. Jolivet. Group antenatal care models in low- and middleincome countries: a systematic evidence synthesis. Reprod. Health 15, 38 (2018)

[13] M. Redshaw, K. Heikkila. Delivered with care: a national survey of women's experience of maternity care 2010. National Perinatal Epidemiology Unit, Oxford, November 2010

[14] B. Hatherall, J. Morris, F. Jamal, L. Sweeney, M. Wiggins, I. Kaur, A. Renton, A. Harden. Timing of the initiation of antenatal care: An exploratory qualitative study of women and service providers in East London. Midwifery 36, 1-7 (2016)

[15] C. Mittiga, K. Ettridge, K. Martin, G. Tucker, R. Dubyna, B. Catcheside, W. Scheil, L. Maksimovic. Sociodemographic correlates of smoking in pregnancy and antenatalcare attendance in Indigenous and non-Indigenous women in South Australia. Aust. J. Primary Health 22(5), 452-460 (2015)

[16] Euro-Peristat Project. European Perinatal Health Report. Core indicators of the health and care of pregnant women and babies in Europe in 2015. November 2018, pp. 56-58

[17] V. Flenady, L. Koopmans, P. Middleton, J.F. Frøen, G.C. Smith, K. Gibbons, M. Coory, A. Gordon, D. Ellwood, H.D. McIntyre, R. Fretts, M. Ezzat. Major risk factors for stillbirth in high-income countries: a systematic review and meta-analysis. Lancet 377, 1331-40 (2011)

[18] M. Jansone, G. Lindmark, J. Langhoff-Roos. Perinatal deaths and insufficient antenatal care in Latvia. Acta. Obstet Gynecol. Scand 80(12), 1091-5 (2001)

[19] Republic of Latvia Cabinet Rules No. 259 (06.06.2018) Maternal and Child Health Improvement Plan 2018-2020 